\title{
Toxines et fimbriae produites par les souches Escherichia coli isolées de fèces de veaux diarrhéiques en Algérie
}

\author{
A. Mohamed Ou Said ${ }^{1,4}$ P. Pohl ${ }^{2}$ \\ J. De Rycke ${ }^{3}$ M. Contrepois ${ }^{*}$
}

\author{
Mots-clés \\ Bovin - Veau - Diarrhée - Escherichia \\ coli - Toxicité - Algérie.
}

\begin{abstract}
Résumé
Cent soixante-cinq souches de Escherichia coli isolées dans différents secteurs géographiques (200 km autour d'Alger) de 45 veaux diarrhéiques et de 4 veaux sains ont été étudiées pour la production de cytotoxines (CNF1, CNF2, VT1 et VT2) et de fimbriae (K99, F17a, F17b, F17c, Att111 et CS31A). La plupart des veaux étaient âgés de deux à quatre semaines. Les souches $\mathrm{E}$. coli produisant CNF2 et les fimbriae F17b ou F17c prédominaient dans la région de Ain Défla et de Bordj Bou Arréridj où respectivement 100 et 75 p. 100 de veaux étaient positifs. Dans la proche périphérie d'Alger, des souches $E$. coli non cytotoxinogènes produisant les fimbriae CS31A et (ou) F17c ont été isolées. $D$ ans la région de M édéa ces deux types de souches ont été isolées. Peu de souches E. coli VT+ ont été identifiées. L'étude spécifique des souches cytotoxiques pour la production des fimbriae F17 a montré que 50 p. 100 des souches CNF2 + produisaient F17b ou F17c.
\end{abstract}

\section{INTRODUCTION}

Escherichia coli est une bactérie commune mais certains pathotypes sont associés à des infections intestinales ou septicémiques chez l'homme et l'animal (25). Parmi les principaux facteurs de virulence, on peut citer la production d'entérotoxines (thermostable ou thermolabile) ou de cytotoxines (vérotoxines VT1 ou VT2 et les facteurs nécrosants CNF1 ou CNF2) $(22,35,36)$. L'adhésion aux entérocytes agit en synergie avec les entérotoxines en permettant aux souches entérotoxinogènes (ETEC) de coloniser massivement l'intestin grêle (41). On peut aussi avancer l'hypothèse que l'adhésion aux entérocytes des $E$. coli $\mathrm{VT}^{+}$(VTEC) ou $\mathrm{CNF}^{+}(\mathrm{NTEC}$ pour $\underline{n}$ ecrotoxigenic $\underline{E}$. $\underline{\text { coli }}$ ) favorise la colonisation intestinale et donc augmente la quantité des cytotoxines qui agissent localement ou diffusent dans l'organisme.

Les ETEC du veau ont été étudiées de façon approfondie pour leurs entérotoxines et leurs facteurs de colonisation $(30,40)$. Konowalchuck et coll. (23) ont décrit des souches VTEC parmi les

\footnotetext{
1. Ecole nationale vétérinaire d'Alger, BP 161, El-Harrach 16200, Algérie

2. Institut national de recherches vétérinaires, Groeselenberg 99, B-1180 Bruxelles, Belgique

3. Ecole nationale vétérinaire, Laboratoire associé Inra de Biologie moléculaire, 23 chemin des capelles, 31076 Toulouse, France

4. Inra, Centre de recherches de Clermont Ferrand-Theix, Laboratoire de microbiologie, 63122 Saint-Genès Champanelle, France

* Auteur pour la correspondance :

Tél. : +33 (0)4 73624244 ; fax : +33 (0)4 73624581

E-mail : contrep@clermont.inra.fr
}

isolats d'origine humaine ou animale. Certains Escherichia coli vérotoxinogènes possèdent le gène eae (pour entérocyte attachement effacement) codant pour la protéine intimine, associée avec les propriétés d'adhérence et la capacité de produire des lésions d'attachement-effacement des microvillosités intestinales (21). Ultérieurement ont été identifiés les NTEC chez l'homme (8) puis chez le veau $(5,13)$. Les travaux initiés par De Rycke et coll. (12, 13) et poursuivis par Oswald et coll. (31) ont conduit à distinguer les toxines $\mathrm{CNF} 1$ et $\mathrm{CNF} 2$. La première correspond à la toxine décrite par Caprioli et coll. (8), la seconde est la toxine Vir identifiée par Smith (39). CNF2 est décrite uniquement parmi les souches d'origine bovine ou ovine $(4,13,37)$. Les gènes gouvernant la biogenèse de CNF1 sont chromosomiques et souvent associés à ceux codant pour l'hémolysine $\alpha$ (18) et les fimbriae de type Pap (6), l'ensemble de ces gènes étant regroupés dans un îlot de pathogénicité (43). Au contraire, les gènes codant pour CNF2 sont décrits à ce jour sur un plasmide $\mathrm{pVir}$ en association avec ceux gouvernant la biogenèse des fimbriae F17b (15). A ce groupe de gènes seraient aussi associés ceux codant pour une autre toxine dite CDTIII (pour cytolethal distending toxin de type III) récemment décrite, l'ensemble étant regroupé sur un îlot de pathogénicité du plasmide pVir (33). Les fimbriae F17b sont des facteurs de colonisation de l'intestin de veau et ils appartiennent à la famille des fimbriae F17 dans laquelle on distingue au moins les quatre variants F17a, F17b, F17c et Att111 (2)

Dans cette étude, les auteurs montrent que des souches E. coli produisant CNF2 représentent un des pathotypes majeurs isolé de veaux atteints de diarrhées en Algérie et les fimbriae de la famille F17 produits par les souches E. coli $\mathrm{CNF}^{+}$ont été identifiées. 
MATERIEL ET METHODES

\section{Les souches bactériennes}

Dans une étude antérieure, 492 souches de E. coli isolées de 45 veaux diarrhéiques et de 4 veaux sains provenant de sept zones géographiques correspondant à un périmètre de $200 \mathrm{~km}$ autour d'Alger (tableau I) ont été étudiées. Les caractères recherchés étaient les facteurs de colonisation et de production de colicines, la présence d'aérobactine, d'hémolysine $\alpha$, d'entérohémolysine, les sérogroupes $\mathrm{O}$ et la résistance aux antibiotiques (28). Dans cette étude, environ 10 souches de $E$. coli ont été isolées des fèces de chaque veau. Les caractéristiques des 10 souches et le profil électrophorétique des extraits bactériens obtenus par chauffage à $60{ }^{\circ} \mathrm{C}(42)$, migration en gel de polyacrylamide dans des conditions dénaturantes et coloration à l'argent, ont permis de sélectionner 1 à 4 souches représentatives des 10 souches de la flore colibacillaire dominante de chaque veau. Les 165 souches ainsi sélectionnées ont été conservées sur milieu Dorset-egg à $+4{ }^{\circ} \mathrm{C}$. Elles ont fait l'objet dans ce travail d'une étude spécifique pour la production des cytotoxines VT et CNF, des facteurs de colonisation K99, F17a, F17b, F17c, Att111 et CS31A et la présence du gène eae. Les souches de référence pour les différents facteurs de virulence sont indiquées dans le tableau II.

\section{Recherche des cytotoxines et du gène eae}

\section{Hybridation avec des sondes $A D N$ spécifiques} pour VT1, VT2, CNF1, CNF2 et EAE

L'hybridation sur colonies a été effectuée selon les méthodes décrites par Mainil et coll. (27) et Oswald et coll. (32). Les sondes ADN provenaient des souches $E$. coli $\mathrm{H} 30, \mathrm{~B} 2 \mathrm{~F} 1 / 3$, BM2-1, S5 et E2348/69 respectivement pour les gènes correspondant à VT1, VT2, CNF1, CNF2 et EAE. Après transfert sur papier filtre (Whatman 541, Whatman Inc. Pleuger, Belgique), les cellules ont été lysées, l'ADN dénaturé et l'hybridation des sondes s'est faite pendant une nuit à $65^{\circ} \mathrm{C}$. Après lavage, les filtres ont été autoradiographiés pendant 1 à 2 jours.

\section{Cytotoxicité sur des cellules en culture}

Les souches $E$. coli ont été cultivées à $37^{\circ} \mathrm{C}$ pendant $18 \mathrm{~h}$ sur bouillon trypticase soja (Difco). Après centrifugation $(10000 \mathrm{~g}$, $30 \mathrm{~min})$, les bactéries en PBS $\left(10^{10}\right.$ cellules/ml) ont été lysées par sonification à $4{ }^{\circ} \mathrm{C}$ (MSE ultrasonic disintegrator, Crawley, UK). Les lysats ont été centrifugés (10 $000 \mathrm{~g}, 15 \mathrm{~min})$ et le surnageant filtré à $0,22 \mu \mathrm{m}$ a été testé sur les cellules HeLa en culture (13). Les toxines VT ont produit la lyse des cellules, alors que les toxines $\mathrm{CNF}$ ont induit une multinucléation des cellules.

\section{Toxicité sur souris}

Les lysats stériles obtenus selon la procédure décrite précédemment ont été injectés par voie intrapéritonéale à des souris âgées de 7 à 8 semaines $(0,5 \mathrm{ml} /$ souris $)$. Après observation des souris pendant une semaine les lysats ont été considérés toxiques lorsqu'au moins 3 souris sur 5 sont mortes. La mortalité était le plus souvent de 5 souris sur 5. La toxicité sur souris a été utilisée pour valider ce test pour les laboratoires qui ne disposent pas de cellules en culture ou ne peuvent pas utiliser les méthodes moléculaires.

\section{Identification des fimbriae}

\section{Méthodes biochimiques et immunologiques}

Les souches ont été cultivées sur Minca gélosé (20) supplémenté avec 0,2 p. 100 de glucose pendant $18 \mathrm{~h}$ à $37^{\circ} \mathrm{C}$. Les bactéries à la
Tableau I

Age et répartition géographique des veaux

\begin{tabular}{lcccc}
$\begin{array}{l}\text { Secteur } \\
\text { géographique }\end{array}$ & \multicolumn{3}{c}{ Age des veaux } & Total \\
\cline { 2 - 4 } & $\mathbf{4}$ à 15 & $\mathbf{1 6}$ à $\mathbf{3 0}$ & $\mathbf{3 1}$ à $\mathbf{4 5}$ & \\
\cline { 1 - 4 } Ain Défla & & 8 & 3 & 11 \\
M édéa & 1 & 9 & 1 & 11 \\
Alger & 2 & 2 & & 4 \\
Bordj Bou Arréridj & & 3 & 1 & 4 \\
Bouira & & 3 & & 3 \\
Tipaza & 2 & 6 & & 8 \\
(1) & 1 & 3 & 1 & 4 \\
Béjaia & & 3 & & 4 \\
Total & 6 & 37 & 6 & 49
\end{tabular}

(1) Veaux sains

Tableau II

Souches de Escherichia coli de référence utilisées pour cette étude

\begin{tabular}{|c|c|c|}
\hline $\begin{array}{l}\text { Nom de } \\
\text { la souche }\end{array}$ & $\begin{array}{l}\text { Facteurs de viru- } \\
\text { lence et fimbriae }\end{array}$ & Références \\
\hline K12 (HB101) & Contrôle négatif & $\begin{array}{l}\text { Davis et coll., } \\
1982\end{array}$ \\
\hline H30 & VT1 & $\begin{array}{l}\text { Konowalchuck et coll., } \\
1977\end{array}$ \\
\hline $\mathrm{B} 2 \mathrm{~F} 1 / 3$ & VT1 + VT2 & $\begin{array}{l}\text { Scotland et coll., } \\
1985\end{array}$ \\
\hline BM 2-1 & CNF1 & $\begin{array}{l}\text { De Rycke et coll., } \\
1990\end{array}$ \\
\hline H209 Virt & CNF2, F17b & $\begin{array}{l}\text { Smith, } 1974 \\
\text { El-M azouari et coll., } \\
1994\end{array}$ \\
\hline S5 & CNF2, F17b & Smith, 1974 \\
\hline B41 & K99, STa & $\varnothing$ rskov et coll., 1975 \\
\hline $25 \mathrm{KH} 9$ & $\mathrm{~F} 17 \mathrm{a}$ & $\begin{array}{l}\text { Pohl et coll., } 1982 \\
\text { Lintermans et coll., } \\
1988\end{array}$ \\
\hline $111 \mathrm{KH} 86$ & Att111 & Bertels et coll., 1989 \\
\hline $31 \mathrm{~A}$ & CS31A & $\begin{array}{l}\text { Girardeau et coll., } \\
1988\end{array}$ \\
\hline $31 \mathrm{~A}$ & F17c & Bertin et coll., 1996a \\
\hline E2348/69 & EAE & Jerse et coll., 1990 \\
\hline
\end{tabular}

surface de la gélose dans une boîte de Pétri ont été collectées dans $1 \mathrm{ml}$ d'une solution de $\mathrm{NaCl}$ à $8,5 \mathrm{~g} / \mathrm{l}$ et chauffées $20 \mathrm{~min}$ à $60^{\circ} \mathrm{C}$ (42). Après centrifugation ( $7000 \mathrm{~g}, 10 \mathrm{~min})$, le surnageant a été soumis à une électrophorèse en conditions dénaturantes (24). Après migration, le gel de polyacrylamide a été coloré à l'argent (29). Les sous-unités des fimbriae avaient des masses moléculaires 
apparentes de 18, 18,5 et $29 \mathrm{kDa}$ respectivement pour Att111, K99 et CS31A. Les sous-unités pour F17a, F17b et F17c avaient des poids moléculaires apparents de $20 \mathrm{kDa}$. L'identification des fimbriae a été complétée par des méthodes immunologiques en utilisant des antisérums spécifiques des différents fimbriae. L'agglutination des bactéries sur lame a été utilisée pour K99, Att111 et F17a. Une méthode immunodot utilisant les extraits bactériens obtenus par chauffage à $60{ }^{\circ} \mathrm{C}$ a été mise en œuvre pour identifier F17b, F17c et CS31A $(10,19)$.

\section{Identification des gènes correspondant aux pilines et aux adhésines des fimbriae F17}

La méthode PCR utilisée était celle proposée par Bertin et coll. (3). L'électrophorèse en gel d'agarose des produits de la réaction PCR ont fourni des bandes correspondant à 322, 326, 419 et 242 paires de bases respectivement pour les gènes $f 17 a, f 17 b, f 17 c$ et f111-1. Les amorces pour les gènes des adhésines des sous-familles I $(f 17 a-G, f 111-G)$ ou II $(f 17 b-G, f 17 c-G)$ ont conduit à l'amplification de segments de 618 paires de bases.

\section{RESU LTATS}

\section{Souches de Escherichia coli cytotoxinogènes et présence du gène eae}

Cinquante et une souche (31 p. 100) étaient positives pour la toxicité sur souris, la cytotoxicité sur cellules HeLa et l'hybridation avec les sondes, la corrélation étant de 100 p. 100 pour les trois méthodes (tableau III). L'hybridation avec les sondes indique qu'aucune souche $E$. coli n'a produit CNF1 mais que les souches $\mathrm{CNF}^{+}{ }^{+}$ont prédominé (84 p. 100 des souches cytotoxiques). Treize souches ont produit VT1 ou VT2 en association ou non avec CNF2. Les souches $E$. coli cytotoxiques ont été isolées chez 53 p. 100 des veaux mais avec une fréquence différente selon les secteurs géographiques. Les souches de $E$. coli $\mathrm{CNF}^{+}$ont été isolées en grand nombre pour les onze veaux étudiés près de Ain Défla (100 p. 100 des veaux positifs) et pour trois des quatre veaux de la région de Bordj Bou Arréridj. Des souches E. coli $\mathrm{CNF}^{+}$ont aussi été isolées chez plusieurs veaux $(5 / 11)$ du secteur de Médéa. Une souche $\mathrm{CNF}^{+}$a été isolée chez un veau sain de la région de Tipaza. Cinq souches provenant de trois veaux étaient $\mathrm{VT}^{+}$et $\mathrm{CNF}^{+}$alors que huit autres souches provenant de six veaux étaient $\mathrm{VT}^{+}$seulement. A l'exception de quatre souches isolées chez un veau de 15 jours, toutes les souches $\mathrm{CNF}^{+}$provenaient de veaux âgés de 20 à 45 jours. Les souches $\mathrm{VT}^{+}$ont été isolées chez les veaux de 15 à 21 jours. Parmi les cinq souches possédant le gène eae, une seulement produisait une cytotoxine (VT1).

\section{Identification des fimbriae}

Des fimbriae ont été identifiées pour 60 des souches (36 p. 100). Aucune souche ne produisait F17a. Deux souches de E. coli K99 ${ }^{+}$ ont été identifiées chez un veau de quatre jours dans la région de Tipaza. Att111 a été trouvé chez deux souches non cytotoxinogènes d'un veau de la région de Bouira. CS31A a été identifié seul ou en association avec F17c chez six souches de E. coli non toxinogènes provenant de deux veaux de la région d'Alger et neuf souches chez six veaux de la région de Médéa. F17b a été identifié exclusivement chez des souches E. coli $\mathrm{CNF}^{+}$des veaux de Ain Défla et de Médéa. F17c était produit par 20 des 165 souches étudiées dont 5 positives pour $\mathrm{CNF}^{+}$.

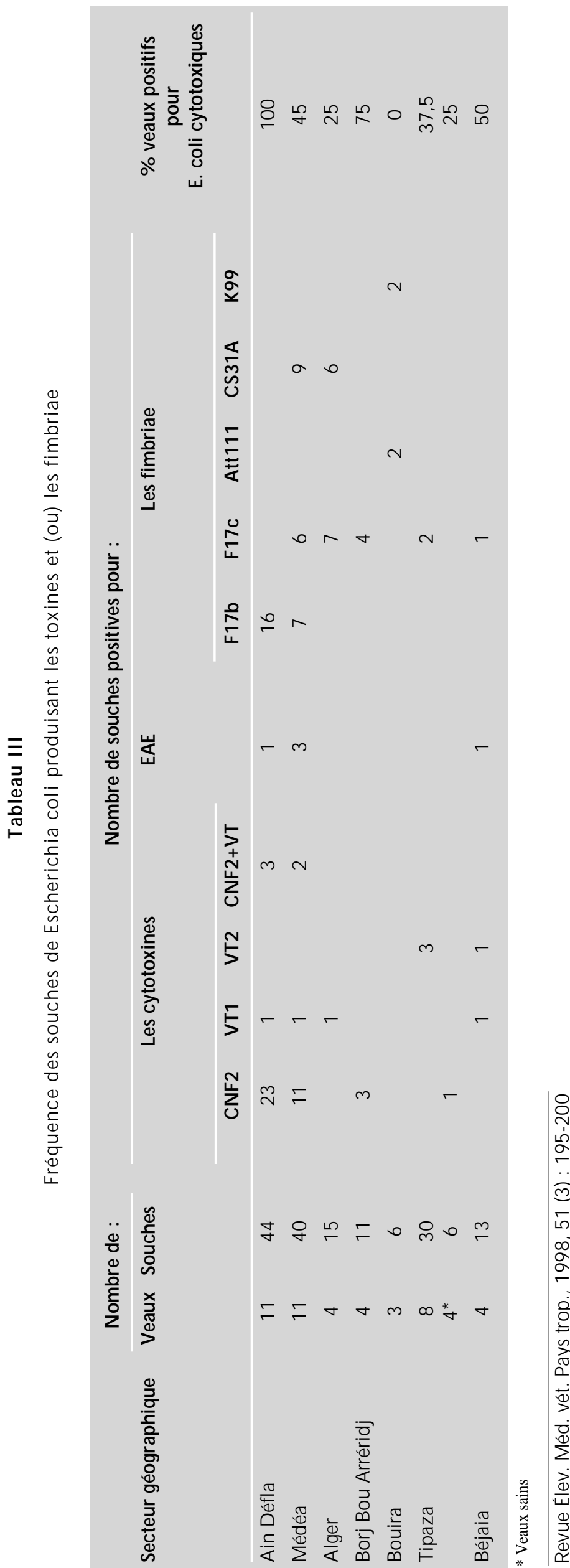


L'identification génotypique pour les pilines et les adhésines de la famille F17 a été conduite exclusivement che 2 les 51 souches cytotoxiques afin de confirmer les résultats de l'étude phénotypique (tableau IV). Trente pour cent des 53 souches CNF2+ étaient positives pour les pilines ou les adhésines de la famille F17, alors que la majorité des souches $\mathrm{VT}^{+}(77 \mathrm{p}$. 100) étaient négatives pour les gènes de la famille F17. Pour 23 souches il s'agissait des gènes codant pour les fimbriae F17b et pour 6 souches, pour les fimbriae F17c et dans tous les cas associés aux gènes des adhésines de la sous-famille II définie par Bertin et coll. (3). Une trentième souche $\mathrm{CNF}^{+}$était positive seulement pour les gènes des adhésines de la sous-famille II. L'étude électrophorétique des souches $E$, coli $\mathrm{F} 17 \mathrm{~b}^{+}$a mis en évidence une hétérogénéité pour le poids moléculaire apparent (environ $20 \mathrm{kDa}$ ) des sous-unités F17b-A (figure 1).

\section{DISCUSSION ET CONCLUSION}

Ces travaux sont un élément d'une étude plus globale visant à préciser l'étiologie infectieuse des diarrhées rencontrées chez les veaux en Algérie, incluant la recherche des virus et parasites. L'étude spécifique des colibacilles entéropathogènes n'a mis en évidence des colibacilles entérotoxinogènes $\mathrm{K} 99^{+}$que chez un veau âgé de quatre jours. Il s'agit d'un cas très particulier puisque le veau était issu d'une vache importée d'Europe en fin de gestation. La majorité des autres veaux diarrhéiques étaient plus âgés, la plupart ayant doux à quatre semaines. L'étude a permis d'identifier plusieurs secteurs géographiques où dominaient certains pathotypes. Dans la région de Ain Défla ont été identifiées majoritairement des souches $E$. coli $\mathrm{CNF}_{2}^{+}, \mathrm{F} 17 \mathrm{~b}^{+}$et dans celle de Bordj Bou Arréridj des souches $\mathrm{CNF}^{+}, \mathrm{F} 17 \mathrm{c}$. Dans la périphérie d'Alger, les souches $E$. coli produisaient CS31A et F17c. Dans la région de Médéa ont été isolées des souches $\mathrm{CNF}^{+}, \mathrm{F} 17 \mathrm{~b}$ ou CS31A et F17c. En revanche, si 13 souches $E$. coli $\mathrm{VT}^{+}$ont été identifiées $(7,8$ p. 100$)$ dont 5 associant la production des cytotoxines CNF2 et VT, ces isolats provenaient de veaux répartis dans les différentes zones géographiques.

Les $E$. coli $\mathrm{CNF}^{+}$ont été associés aux diarrhées et septicémies chez l'agneau (12) et le veau (37). Par ailleurs, l'infection expérimentale du porcelet par des souches $E$. coli produisant les toxines $\mathrm{CNF}(17,44)$ indique que les cytotoxines CNF contribuent à la pathogénicité des $E$. coli dans la colibacillose du porc. Toutefois, différentes études montrent qu'on isole $E$. coli $\mathrm{CNF}^{+}$avec une fréquence comparable chez les animaux malades ou sains $(5,7,35)$ el, dans cetle étude, l'échantillonnage des quatre veaux sains ne permet pas une comparaison significative avec les animaux malades.

La production de VT est fréquente pour les souches $E$. coli isolées dc bovins malades ou sains, mais ce sont seulement les souches $\mathrm{VT}^{+}$produisant l'intimine (souches $\mathrm{EAE}^{+}$) et donc les lésions d'attachement-effacement qui seraient pathogènes (34). Ce n'était pas le cas pour la majorité des souches $\mathrm{VT}^{+}$identifiées au cours de cette étude puisqu'une seule souche possédait le gène eae. Pour l'autre pathotype correspondant aux souches $E$. coli $\mathrm{CS}_{31 A^{+}}$, ce caractère est corrélé à des propriétés septicémiques (10) ou à un syndrome de gastro-entérite avec des signes d'alaxie (16). E. coli CS31A ${ }^{+}$se classe dans la famille des bactéries septicémiques pathogènes opportunistes.

L'étude des fimbriae des souches $E$. coli $\mathrm{CNF}^{+}$confirme que la majorité d'cntrc clles produisent les fimbriae F17b (15) connues également sous l'appellation de Vir (39), mais certaines expriment le variant $\mathrm{F} 17 \mathrm{c}$ comme cela avait été mentionné par Bertin et coll. (3). Les légères différences dans les poids moléculaires apparents

\section{Tableau IV}

Gènes gouvernant la biogenèse des pilines ou des adhésines de la famille F17 parmi les souches de $E$. coli cytotoxiques

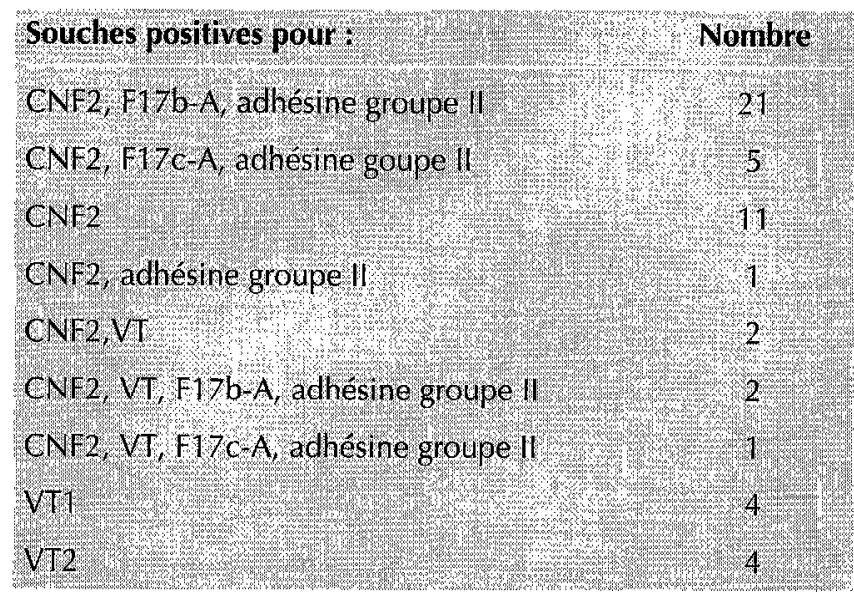

\section{6}

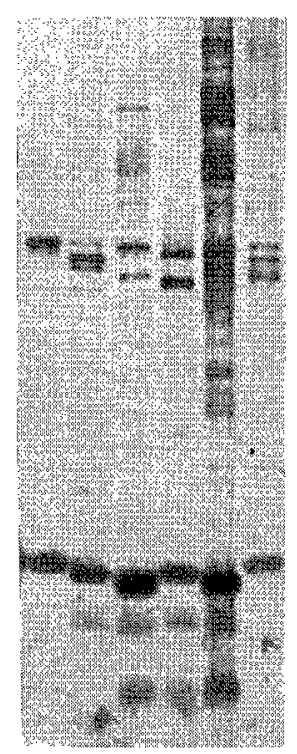

$\leftarrow 20 \mathrm{kDa}$

Figure 1 : Profil électrophorétique des souches bactériennes 1. Souche de Escherichia coli H209 F17b+, référence pour F17b 2. Souche de Escherichia coli $H C 22$, variant F1 7b, type 1

3. Souche de Escherichia coli $A D 52$, variant $F 17 b$, type 2

4. Souche de Escherichia coli $A D 16$, variant $F 17 b$, type 1

5. Souche de Escherichia coli $A D 1 a$, variant $F 17 b$, type 2

6. Souche de Escherichia coli S5, référence pour F17b.

des sous-unités de F17b (figure 1) suggèrent une certaine hétérogénéité des fimbriae F17b. On peut penser qu'une petite différence des séquences en acides aminés des sous-unités explique la diversité des profils électrophorétiques comme cela a déjà été noté pour d'autres fimbriac $(9,14)$. Enfin, unc ćtude complémentaire montre que les sérotypes $\mathrm{O} 3: \mathrm{K} ?: \mathrm{H} 19$ et $\mathrm{O} 55: \mathrm{K}^{-}: \mathrm{H} 32$ sont largement représentés pour la famille des souches $\mathrm{CNF} 2, \mathrm{~F} 17 \mathrm{~b}^{+}$, alors que les souches $\mathrm{CNF} 2, \mathrm{~F} 17 \mathrm{c}^{+}$ont pour sérotype $\mathrm{O} 115: \mathrm{K}$ ?:H10. 
En conclusion, si cette étude apporte des précisions intéressantes sur les caractéristiques des colibacilles cytotoxiques, elle se proposait en premier lieu de préciser l'étiologie colibacillaire des pathologies diarrhéiques chez les veaux en Algérie. Les résultats obtenus ne sont pas complètement démonstratifs. Ils mettent en exergue des cytotoxines et des facteurs de colonisation dont les effets in vitro sont bien documentés. Toutefois, différents résultats épidémiologiques publiés à ce jour montrent que l'isolement de colibacilles $\mathrm{CNF}^{+}$ne différencie pas clairement les animaux malades des animaux sains $(5,35)$. De même, les bovins sont porteurs asymptomatiques pour les souches $E$. coli $\mathrm{VT}^{+}$, le pouvoir pathogène s'exprimant seulement en association avec le caractère $\mathrm{EAE}^{+}$(34). Par ailleurs, les colibacilles $\mathrm{CS}_{3} 1 \mathrm{~A}^{+}$sont des pathogènes opportunistes (10). Si on ne peut pas écarter le rôle de ces colibacilles dans les pathologies diarrhéiques, d'autres caractères complémentaires plus discriminants restent à identifier.

\section{Remerciements}

Ce travail est dédié au Pr Jacques Sevestre de l'Env de Nantes. A. Mohamed Ou Said remercie le Conseil régional des Pays-de-Loire pour son aide financière. Nous remercions M. Marin de l'Inrv de Bruxelles ainsi que C. Tasca de l'Env de Toulouse pour leur assistance technique.

\section{BIBLIO GRAPHIE}

1. BERTELS A., POHL P., SCHLICKER F., VAN DRIESSCHE E., CHARLIER G., DE GREVE H., LINTERMANS P., 1989. Isolatie van het Att111 fimbrieel antigen op Escherichia coli geisoleerd uit kalverdiarree: karakterizatie en evaluatie van de noodzaak tot aanpassing van de vaccins ter bestrijding van neonatale colidiarree. Vlaams Diergeneeskd. Tijdschr., 58: 18-122.

2. BERTIN Y., GIRARDEAU J.P., DARFEUILLE-MICHAUD A., CONTREPOIS M., 1996a. Characterisation of 20K-fimbriae, a new adhesin of septicemic and diarrhea-associated Escherichia coli strains, that belongs to a family of adhesins with $\mathrm{N}$-acetyl-D-glucosamine recognition. Infect. Immun., 64: 332-342.

3. BERTIN Y., MARTIN C., OSWALD E., GIRARDEAU J.P., 1996b. Rapid and specific detection of F17-related pilin and adhesin genes in diarrheic and septicemic Escherichia coli strains by multiplex polymerase chain reaction. J. clin. Microbiol., 34: 2921-2928.

4. BLANCO M., BLANCO J., BLANCO J.E., RAMOS J., 1993. Enterotoxigenic, verotoxigenic and necrotoxigenic Escherichia coli isolated from cattle in Spain. Am. J. vet. Res., 54: 1446-1451.

5. BLANCO J., GONZALEZ E.A., BLANCO M., REGEIRO B., BERNADEZ I., 1988. Production of toxins by Escherichia coli strains isolated from calves with diarrhoea in Galicia (north-western Spain). Vet. Microbiol., 18: 297-311.

6. BLUM G., FALBO V., CAPRIOLI A., HACKER J., 1995. Gene clusters encoding the cytotoxing necrotizing factor type 1 Prs-fimbriae and $\alpha$ hemolysin from the pathogenicity island II of the uropathogenic Escherichia coli strain J96. FEMS Microbiol. Lett., 126: 189-196.

7. BURNS A.L., BALL H.J., FINLAY D.A., 1996. CNF producing Escherichia coli isolated from cattle in Northern Irland. Vet. Microbiol., 49: 235-241.

8. CAPRIOLI A., FALBO F., RODA G.L., RUGGERI F.M., ZONA C., 1983. Partial purification and characterization of an Escherichia coli toxic factor that induces morphological cell alterations. Infect. Immun., 39: 1300-1306

9. CONTREPOIS M., BERTIN Y., GIRARDEAU J.P., PICARD B., GOULET P., 1993. Clonal relationships among bovine pathogenic Escherichia coli producing surface antigen CS31A. FEM S M icrobiol. Lett., 106: 217-222.
10. CONTREPOIS M., DUBOURGUIER H.C., PARODI A.L., GIRARDEAU J.P., OLLIER J.L., 1986. Septicaemic Escherichia coli and experimental infection of calves. Vet. Microbiol., 12: 109-118.

11. DAVIS R.W., BOSTEIN D., ROTH J.R., 1982. Advanced bacterial genetics. Cold Spring Harbor, NY, USA, Cold Spring H arbor Laboratory.

12. DE RYCKE J., GONZALES E.A., BLANCO J., O SWALD E., BO IVIN, R., 1990. Evidence of two types of cytotoxic necrotizing factor (CNF1 and CNF2) in human and animal clinical isolates of Escherichia coli. 0J. Clin. Microbiol., 28: 694-699.

13. DE RYCKE J., GUILLOT J.F., BOIVIN R., 1987. Cytotoxins in non enterotoxigenic strains of Escherichia coli isolated from feces of diarrheic calves. Vet. Microbiol., 15: 137-157.

14. DI MARTINO P., BERTIN Y., GIRARDEAU J.P., LIVRELLI V., JO LY B., DARFEUILLE-MICHAUD A., 1995. Molecular characterization and adhesive properties of CF29K an adhesin of Klebsiella pneumoniae strains involved in nocosomial infections. Infect. Immun., 63 4226-4344.

15. EL-M AZO U ARI K., O SW ALD E., HERN ALSTEENS J.P., LINTERM ANS P., DE GREVE H., 1994. F17-like fimbriae from an invasive Escherichia coli strain producing cytotoxic necrotizing factor type 2 toxin. Infect. Immun., 62: 2633-2638.

16. ESPINASSE J., NAVETAT $H_{\text {., }}$ CONTREPOIS M., BAROUX D., SCHELCHER F., 1991. A new diarrheic syndrome with ataxia in young charolais calves: clinical and microbiological studies. Vet. Rec., $\mathbf{1 2 8}$ 422-425.

17. FAIRBROTHER J.M., NGELKA M., 1994. Extraintestinal Escherichia coli infections in pigs. In: Gyles C.L. Ed., Escherichia coli in domestic animals and man. Oxford, UK, CAB international, p. 221-267.

18. FALBO V., PACE T., PICCI L., PIZZI E., CAPRIO LI A., 1993. Isolation and nucleotide sequence of the gene encoding cytotoxic necrotizing factor 1 of Escherichia coli. Infect. Immun., 61: 4909-4914.

19. GIRARDEAU J.P., DER VARTANIAN M., OLLIER J.L., CONTREPOIS M., 1988. CS31A, a new K88-related fimbrial antigen on bovine enterotoxigenic and septicemic Escherichia coli strains. Infect. Immun., 56: $2180-2188$

20. GUINEE P.A.M., JAN SEN W.H., AGTERBERG C.M., 1976. Detection of the $\mathrm{K} 99$ antigen by means of agglutination and immuno electrophoresis in Escherichia coli isolated from calves and its correlation with enterotoxigenicity. Infect. Immun., 13: 1369-1377.

21. JERSE A.E., YU J., TALL B.D., KAPER J.B., 1990. A genetic locus of enteropathogenic Escherichia coli necessary for the production of attaching and effacing lesions on tissue culture cells. Proc. Natl. Acad. Sci. USA, 87: 7839-7843.

22. KARMALI M.A., 1989. Infection by verocytotoxin-producing Escherichia coli. Clin. Microbiol. Rev., 2: 15-38.

23. KO N O W ALCHUK J., SPEIRS J.L., STAVRICS S., 1977. Vero response to a cytotoxin of Escherichia coli. Infect. Immun., 18: 775-779.

24. LAEMMLI U.K., 1970. Cleavage of structural protein during the assembly of the head of bacteriophage T4. Nature (London), 227: 680-685.

25. LEVINE M.M., 1987. Escherichia coli that cause diarrhea: enterotoxigenic, enteropathogenic, enteroinvasive, enterohemorrhagic and enteroadherent. J. infect. Dis., 55: 377-389.

26. LINTERMANS P., POHL P., DEBOECK F., BERTELS A., SCHLICKER C., VANDEKERCKHOVE J., VAN DAMME J., VAN MONTAGU M., DE GREVE H., 1988. Isolation and nucleotide sequence of F17-A gene encoding the structural protein of the F17 fimbriae in bovine enterotoxigenic Escherichia coli. Infect. Immun., 56: 1475-1484.

27. MAINIL J., BEX F., JAQUEMIN E., POHL P., COUTURIER M., KAECKEN BEECK A., 1990. Prevalence of four enterotoxin (STaP, STaH, $\mathrm{STb}$ and LT) and four adhesin subunit (K99, K88, 987P and F41) genes among Escherichia coli isolates from cattle. Am. J. vet. Res., 51 187-190. 
28. MOHAMED OU SAID A., CONTREPOIS M. DER VARTANIAN M. GIRARDEAU J.P., 1994. Facteurs et marqueurs de virulence de souches Escherichia coli isolées de diarrhées chez des veaux âgés de 4 à 45 jours en Algérie. Revue Elev. M éd. vét. Pays trop., 47 : 169-175.

29. OAKLEY B.R., KIRSCH D.R., MORRIS N.E., 1980. A simplified ultrasensitive silver stain for detecting protein in polyacrylamide gels. Anal. Biochem., 105: 361-365.

30. ORSKOV F., ORSKOV I., SMITH H.W., WILLIAMS H., SOJKA W.J., 1975. The establishment of K99, a thermolabile transmissible Escherichia coli K antigen, previously called "Kco" possessed by calf and lamb enteropathogenic strains. Acta Pathol. Microbiol. Scand., 83 31-36.

31. OSWALD E., DE RYCKE J., LINTERMANS P., VAN MUYLEM K., MAIN IL J., DAU BE G., POHL P., 1991. Virulence factors associated with the cytotoxic necrotizing factor type two (CNF2) in bovine diarrheic and septicemic strains of Escherichia coli. J. clin. Microbiol., 29: 2522-2527.

32. OSWALD E., POHL P., JACQUEMIN E., LINTERMANS P., VAN MUYLEM K., O'BRIEN A. D., 1994. Specific DNA probes to detect Escherichia coli strains producing cytotoxic necrotizing factor type 1 or type 2. J. Med. Microbiol., 40: 428-434.

33. PERES S.Y., MARCHES O., DAIGLE F., NOUGAYREDE J.P., HERAULT F., TASCA C., DE RYCKE J., OSWALD E., 1997. A new cytolethal distending toxin (CDT) from Escherichia coli producing CNF2 blocks HeLa cell division in G2/M phase. Mol. Microbiol., 24: 1095-1107.

34. POHL P., 1991. Les Escherichia coli verotoxinogènes isolés des bovins. Ann. Méd. vét., 135 : 569-576.

35. POHL P., IMBERECHTS M., MARIN M., SCHLINCKER C., STOCKMANS F., 1997. Prévalence des gènes codant pour les cytotoxines nécrosantes (CNF1 et CNF2) chez les Escherichia coli isolés de bovins malades ou asymptomatiques. Ann. Med. vet., 141: 161-164.
36. POHL P., LINTERMANS P., VAN MUYLEN K., SCHOTTE M., 1982. Colibacilles entérotoxinogènes de veau possédant un antigène d'attachement différent de l'antigène K99. Ann. Méd. vét., 126 : 569-571.

37. POHL P., OSWALD E., VAN MUYLEN K., JACQUEMIN E., LINTERM ANS P., MAINIL J., 1993. Escherichia coli producing CNF1 et CNF2 cytotoxins in animals with different disorders. Vet. Res., 24: 311-315.

38. SCOTLAND S.M., SMITH H.R., ROWE B., 1985. Two distinct toxins active on vero cells from Escherichia coli 0 157. Lancet, 19: 885-886.

39. SM ITH H.W., 1974. A search for transmissible characters in invasive strains of Escherichia coli: the discovery of a plasmid-controlled toxin and a plasmid-controlled lethal character closely associated, or identical, with colicine V. J. gen. Microbiol., 83: 95-111.

40. SMITH H.W., HALLS S., 1967. Studies on Escherichia coli enterotoxin. J. Pathol. Bacteriol., 93: 531-543.

41. SMITH H.W., HUGGINS M.B., 1979. Experimental infections of calves piglets and lambs with mixture of invasive and enteropathogenic strains of Escherichia coli. J. Med. Microbiol., 12: 507-510.

42. STIRM S., ORSKOV F., 1967. Episome carried surface antigen K88 of E. coli II. Isolation and chemical analysis. J. Bacteriol., 143: 731-739.

43. SWENSON D.L., BUKANOV N.O., BERG D.E., WELCH R.A., 1996. Two pathogenicity islands in uropathogenic Escherichia coli J96: Cosmid cloning and sample sequencing. Infect. Immun., 64: 3736-3743.

44. WRAY C., PIERCY D.W.T., COOLEY W.A., 1993. Experimental infection of neonatal pigs with CNF toxin-producing strains of Escherichia coli. Res. vet. Sci., 54: 290-298.

Reçu le 26.3.98, accepté le 18.11 .98

\section{Summary}

Mohamed O u Said A., Pohl P., De Rycke J., Contrepois M Toxins and fimbriae produced by strains of Escherichia coli isolated from feces of diarrheic calves in Algeria

O ne hundred and sixty-five Escherichia coli strains isolated from several geographic areas (200 km around Algiers) from 45 diarrheic and 4 healthy calves were studied for cytotoxins (CNF1, CNF2, VT1, VT2) and fimbrial antigens (K99, F17a, F17b, F17c, Att111 and CS31A). Most of the calves were 2 to 4 weeks old. E. coli strains producing CNF2 and F17b or F17c fimbrial antigens were highly prevalent in the Ain Defla and Bordj Bou Arreridj areas with 100 and $75 \%$ of positive calves, respectively. In suburban Algiers, non-cytotoxic E. coli strains producing CS31A and/or F17C surface antigen(s) were isolated. In the Medea area both strain types were isolated. Only few VT-producing E. coli strains were identified. A specific study focusing on F17-related fimbriae produced by cytotoxin-producing E. coli strains showed that $50 \%$ of CNF2 producing isolates were $\mathrm{F} 17 \mathrm{~b}$ or F17c positive.

Key words: Cattle - Calf - Diarrhea - Escherichia coli Toxicity - Algeria.

\section{Resumen}

Mohamed O u Said A., Pohl P., De Rycke J., Contrepois M. Toxinas y fimbrias producidas por las cepas de Escherichia coli aisladas en heces de terneros diarreícos en Argelia

Se estudiaron 165 cepas de Escherichia coli, aisladas en diferentes sectores geográficos (200 km alrededor de Argel), a partir de 45 terneros diarreícos y de 4 terneros sanos, con el fin de detectar la producción de citotoxinas (CNF1, CNF2, VT1 y VT2) y de fimbrias (K99, F17a y F17b, F17c, Att111 y CS31A). La mayoría de los terneros tenían entre dos y cuatro semanas de edad. Las cepas de E. coli que produjeron CNF2 y las fimbrias F17b o F17c predominaron en la región de Ain Defla y de Bordj Bou Arreridj, donde, respectivamente, 100 y $75 \%$ de los terneros fueron positivos. En la periferia cercana de Argel, se aislaron las cepas de E. coli no citotoxígenas que produjeron las fimbrias CS31A y/o F17c. En la región de Medea, se aislaron estos dos tipos de cepas. Se identificaron pocas cepas de E. coli $\mathrm{VT}^{+}$. El estudio específico de las cepas citotóxicas para la producción de fimbrias F17 mostró que $50 \%$ de las cepas $\mathrm{CN} \mathrm{F2}^{+}$produjeron $\mathrm{F} 17 \mathrm{~b}$ o $\mathrm{F} 17 \mathrm{C}$.

Palabras clave: Ganado bovino - Ternero - Diarrea - Escherichia coli - Toxicidad - Argelia. 